\title{
Efectos de la actividad física moderada sobre la ingesta alimentaria en presencia de estrés psicológico
}

Saucedo-Villanueva Esthela Guadalupe*, Flores-Sánchez Ana Celene*, Villanueva-Cruz Lucero de los Ángeles*, García-Sánchez Andrea Alejandra*, Cardona-Perales Donnie Helaman*, Martín del Campo-Cervantes Judith**

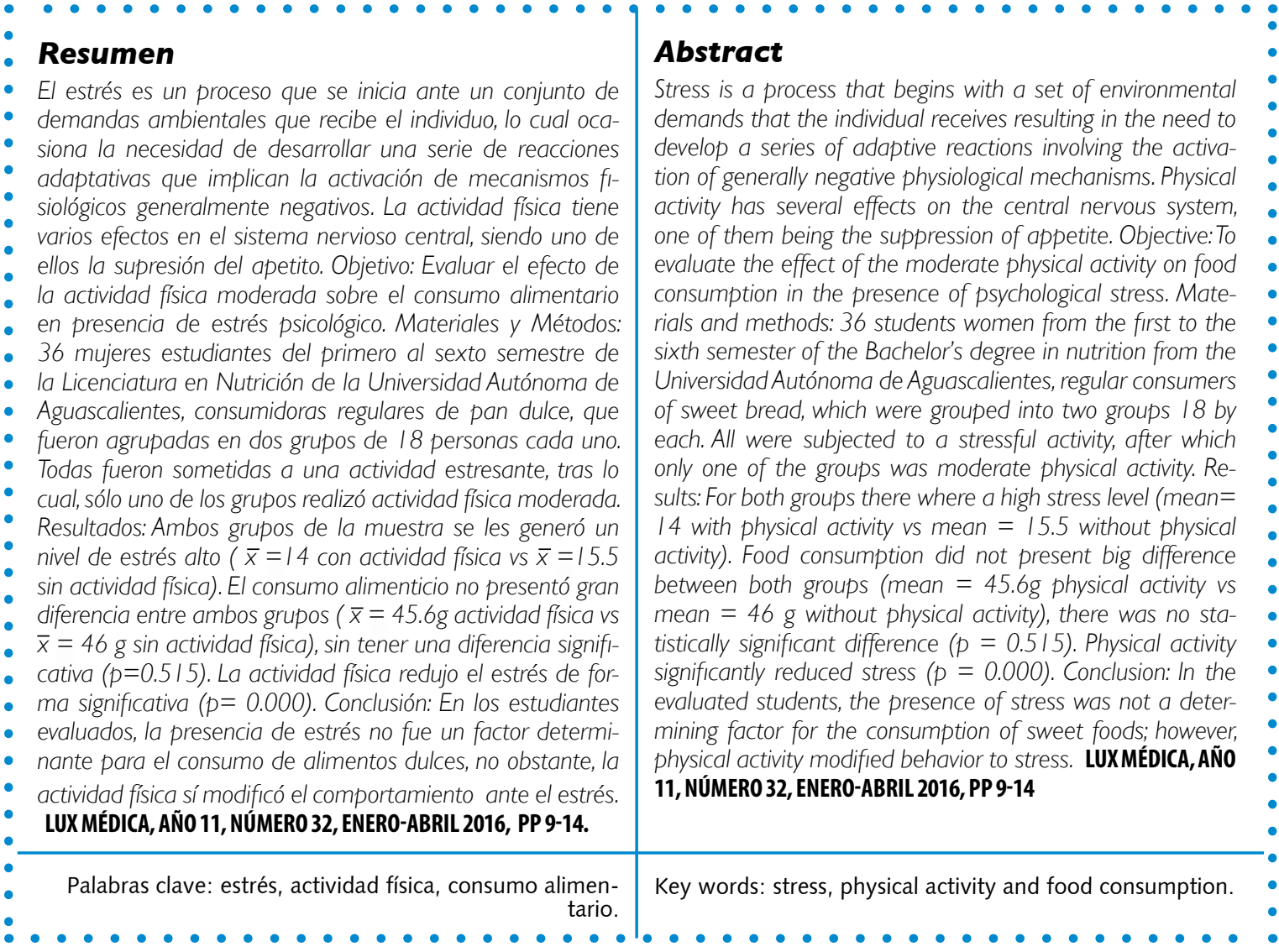

* Estudiantes del séptimo semestre de la Licenciatura en Nutrición del Centro de Ciencias de la Salud de la Universidad Autónoma de Aguascalientes.

** Profesora de tiempo completo, investigadora del Departamento de Nutrición del Centro de Ciencias de la Salud de la Universidad Autónoma de Aguascalientes.

Fecha de recibido: 29 de septiembre 2015

Fecha de aceptación: 9 de febrero 2016

Correspondencia: Judith Martín del Campo, Departamento de Nutrición y Cultura Física, Edificio 107 planta baja. Centro de Ciencias de la Salud, Universidad Autónoma de Aguascalientes. Avenida Universidad número 940 Ciudad Universitaria. Código postal 20131. Aguascalientes, Ags., México. Teléfono 01 (449) 9108443 Correo electrónico jmartic@correo.uaa.mx 


\section{Introducción}

El estrés es un proceso que se inicia ante un conjunto de demandas ambientales que recibe el individuo, a las que debe dar una respuesta adecuada, poniendo en marcha sus recursos de afrontamiento. ${ }^{1-3}$

Actualmente, la demanda del ambiente (laboral, social) es excesiva frente a los recursos de afrontamiento que posee el individuo; esto acarrea cambios tanto en el ámbito psicosocial como en el laboral, con trabajos de mayor demanda, disminuyendo la convivencia y las relaciones interpersonales. Debido al conjunto de estas demandas ambientales y sociales el ser humano se ve con la necesidad de desarrollar una serie de reacciones adaptativas (escogen sus alimentos y comen con rapidez) ${ }^{4,5}$, las cuales implican una activación de mecanismos fisiológicos generalmente negativos que incluye emociones desagradables entre las cuales están la ansiedad, la ira y la depresión que se ven reflejados en el estrés. ${ }^{1,6}$

La actividad física tiene varios efectos en el sistema nervioso central ${ }^{7}$, como la relajación dinámica a través del movimiento, lo cual puede aumentar el nivel de serotonina y de otros mediadores como la dopamina, encargados de la concentración y motivación, y la $\beta$-endorfina ${ }^{8}$, una neurohormona responsable de la sensación de bienestar que aparece en el ejercicio aeróbico suave después de 30 minutos de haberlo iniciado. Estos factores permiten fundamentar los cambios subjetivos que se experimentan entre la actividad y el estado emocional. 1,9,10

El ejercicio físico eleva la temperatura, lo cual no permite el adecuado funcionamiento del hipotálamo, además aumenta hormonas, como la adrenalina, la glucosa y ácidos grasos libres en el suero. Todo esto provoca el efecto de supresión del apetito. ${ }^{4,11} \mathrm{El}$ objetivo de este trabajo fue evaluar el efecto de la actividad física moderada sobre el consumo alimenticio en presencia de estrés psicológico.

\section{| | | | | | | | | | | | | | | | | | | | | | | | | | | | | | | | | | | | | | | | | | | | | | | | | | | | | | | | | | | | | | | | | | | | | | | | | | | | | | | | | | | | | | | | | | | | | | | | | | | | | | | | | | | | | | | | | | | |}

\section{Material y métodos}

Estudio cuasi experimental. El criterio de inclusión fue: mujer de 18-65 años, clínicamente sana y estudiante de la Licenciatura en Nutrición (primero al sexto semestre) de la Universidad Autónoma de Aguascalientes. La muestra fue no probabilística, por conveniencia. Se utilizó una caminadora con cronómetro incluido (Pro-Form 505 CST modelo PFTL50910.1), un cuestionario de selección muestral, la escala de valoración del estado de ánimo (EVEA), recipientes de plástico, pan dulce, tablas de medición de tasa metabólica en reposo o MET y balanza electrónica de precisión (marca Rhino, modelo BAPRE-3 con capacidad máxima de $3 \mathrm{~kg}$ y división máxima de $0.2 \mathrm{~g}$ ). Se implementó el cuestionario de selección muestral al universo elegido (60 personas), obteniendo a 36 estudiantes. Se dividió la muestra en dos grupos de 18 personas (grupo A y grupo B).

En el grupo $A$, se aplicó el cuestionario EVEA en tres tiempos: el primero, cuan- 
do las participantes llegaron al lugar para medir su nivel de estrés inicial; el segundo, después de la película (generador de estrés) para verificar si se indujo; y el tercero, al momento de terminar la actividad física con el fin de corroborar si se redujo; dicha actividad fue una caminata a paso rápido por $15 \mathrm{~min}(6.4 \mathrm{~km} / \mathrm{h}=5 \mathrm{METs})$, que cumple con la recomendación de la Organización Mundial de la Salud de mínimo 10 minutos continuos para generar un beneficio. Después de ello se le asignó a cada una de las participantes un recipiente con pan dulce del cual disponían a libre demanda. El contenido de pan dulce se contabilizó y pesó previamente.

En el grupo B se aplicó el cuestionario EVEA en dos tiempos: el primero, cuando las participantes llegaron al lugar y el segundo, después de la película (generador de estrés); posteriormente, se le asignó a cada una de las participantes un recipiente con pan dulce del cual disponían a libre demanda. El contenido de pan dulce se contabilizó y pesó previamente. Al término de las actividades se midió en gramos la cantidad de pan dulce consumido por cada participante y se compararon las cantidades consumidas del grupo A con las del grupo B. Se realizó la base de datos, aplicando estadística descriptiva en Excel y la analítica en Minitab 17 considerando diferencia significativa una de $p<0.05$ con un nivel de significancia del $95 \%$.

\section{| | | | | | | | | | | | | | | | | | | | | | | | | | | | | | | | | | | | | | | | | | | | | | | | | | | | | | | | | | | | | | | | | | | | | | | | | | | | | | | | | | | | | | | | | | | | | | | | | | | | | | | | | | | |}

\section{Resultados}

La muestra se integró por 36 mujeres estudiantes de primero a sexto semestre de la Licenciatura en Nutrición de la Universidad Autónoma de Aguascalientes, las cuales presentaron una media de edad de 20 años (DE \pm 5.3 años). Para aplicar la actividad de estrés se dividió a las estudiantes en 2 grupos conformados por 18 mujeres (tabla 1 y 2$)$.

\section{Tabla I}

Distribución de alumnas pertenecientes a la muestra, por cada semestre de la Lic. en Nutrición de la Universidad Autónoma de Aguascalientes del grupo A y B

\begin{tabular}{|lccccc|}
\hline $\begin{array}{l}\text { Grupo A } \\
\text { Semestre }\end{array}$ & $\mathbf{n}=$ & $\overline{\mathbf{x}}$ edad & $\begin{array}{l}\text { Grupo B } \\
\text { Semestre }\end{array}$ & $\mathbf{n}=$ & $\overline{\mathbf{x}}$ edad \\
\hline $6^{\circ}$ & 4 & 20.3 & $5^{\circ}$ & 3 & 20.6 \\
\hline $5^{\circ}$ & 2 & 20 & $4^{\circ}$ & 8 & 19.8 \\
\hline $3^{\circ}$ & 2 & 31 & $3^{\circ}$ & 2 & 20 \\
\hline $2^{\circ}$ & 2 & 18.5 & $2^{\circ}$ & 2 & 19 \\
\hline $1^{\circ}$ & 8 & 19 & $1^{\circ}$ & 3 & 19.3 \\
\hline Total & 18 & 21.2 & Total & 18 & 19.8 \\
\hline
\end{tabular}

Ambos grupos fueron sometidos a un estímulo estresante y sólo un grupo realizó actividad física como medida reductora de estrés. En el grupo que realizó actividad física los resultados de EVEA en los datos de la suma de ítems de estrés fueron los siguientes: en promedio, la cifra inicial correspondió a estrés moderado ( $\bar{x}=9.94)$, 
la puntuación después de la actividad estresante fue de estrés alto $(\bar{x}=14)$ y cifras finales: estrés bajo ( $\bar{x}=4.88)$.

En el grupo que no realizó actividad física los resultados de EVEA en los datos de la suma de ítems de estrés fueron los siguientes: Cifras iniciales: estrés moderado $(\bar{x}=12.16)$, cifras después de la actividad estresante: estrés alto $(\bar{x}=15.5)$.

\section{Tabla 2}

Medias de estrés en diferentes etapas de la prueba del grupo A y B

\begin{tabular}{|llll|}
\hline Grupo A & \multicolumn{3}{c|}{ Grupo B } \\
\hline Previo a actividad física & $\bar{x}=9.9$ & Previo a actividad física & $\overline{\mathrm{x}}=12.1$ \\
\hline Después de actividad estresante & $\overline{\mathrm{x}}=14$ & Después de actividad estresante & $\overline{\mathrm{x}}=18$ \\
\hline Post-actividad física & $\overline{\mathrm{x}}=4.8$ & & \\
\hline
\end{tabular}

Se realizó una diferencia de medias del consumo alimenticio de pan dulce posterior a la actividad estresante con o sin actividad física sin obtener una diferencia significati- va en las medias con un valor de $p=0.515$, para la comparación de ambos grupos, y una zona de rechazo de $t=-0.04$, como se observa en la figura 1.

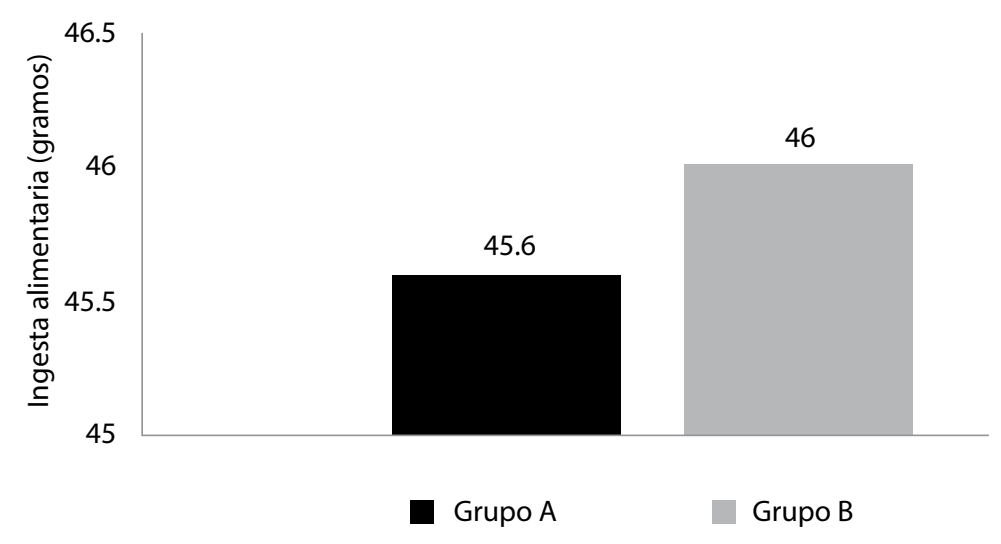

Figura 1. Distribución de la ingesta alimentaria con actividad física y sin actividad física, que presentaron las alumnas de la Lic. en Nutrición de la Universidad Autónoma de Aguascalientes

$N=36$ alumnas, $T$ student $: p=0.515$

El efecto de la actividad física en la reducción del estrés fue significativo al aplicar la comparación de medias entre ambos grupos y obtener un valor de $p=0.000$ y una zona de rechazo de $t=3.80$, como se observa en la figura 2. 


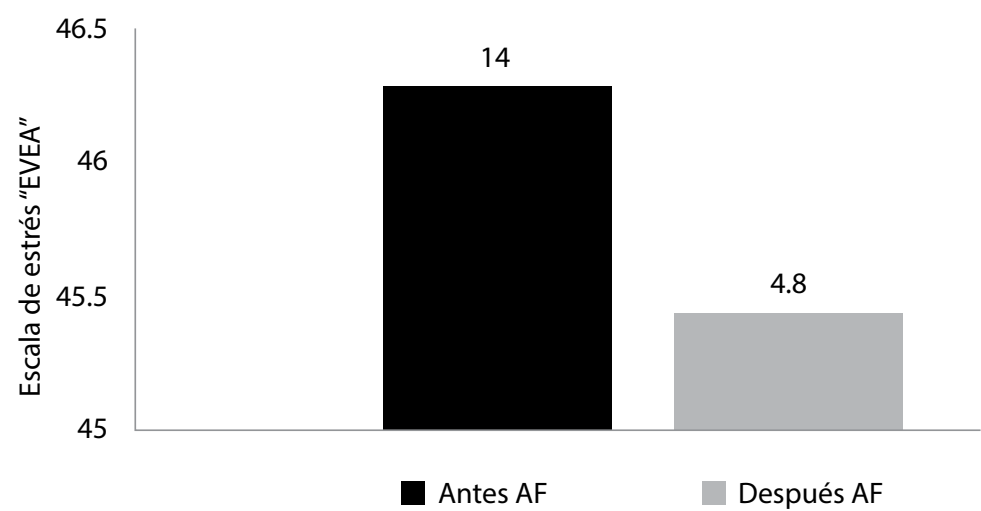

Figura 2. Efecto de la actividad física en la reducción del estrés. $n=36$ alumnas.

EVEA= Escala de Valoración del Estado de Ánimo. AF= Actividad física

\section{Discusión}

La presente investigación tuvo como fin demostrar que la actividad física disminuye el estrés y que, a su vez, el estrés influye en la cantidad de alimento que se ingiere; en este caso el alimento utilizado fue pan dulce de chocolate ${ }^{12,13}$. El resultado obtenido fue que el nivel de estrés no influyó significativamente en el consumo de pan ${ }^{14}$, un resultado semejante fue reportado por Adrián Taylor et al, ${ }^{15}$ quienes concluyeron que la dificultad de la tarea a la que fueron sometidos los participantes no afectó la cantidad de chocolate consumido.

En contraste con la investigación de Taylor en la cual la actividad física se realizó antes de ser sometidos a la actividad estresante, el presente estudio incluyó en primer lugar la actividad estresante en los dos grupos, seguida de la actividad física en uno de los grupos para observar el efecto de la misma sobre el estrés, mientras que Taylor aplicó al primer grupo una actividad física por 15 minutos y en el segundo grupo lo descansaron por el mismo lapso de tiempo. Posteriormente, se subdividió cada grupo en dos para aplicar a uno de ellos una tarea estresante sencilla y al otro una más compleja, mientras se ponía a su disposición el chocolate. A pesar de las diferencias en el tipo de estrés y de alimento, los resultados obtenidos en ambas investigaciones fueron similares. El resultado obtenido en esta investigación fue positivo, al demostrar que la actividad física redujo el estrés de acuerdo a los valores de la escala EVEA (Grupo A: Previo a actividad estresante: $\bar{x}=9.9$, después de actividad estresante: $\bar{x}=14$, post-actividad física: $\bar{x}=4.8$. Grupo B: Previo a actividad estresante: $\bar{x}=12.1$, después de actividad estresante: $\bar{x}=18$ ), como se observa en la tabla $2 .^{16-20}$

En nuestra población se aplicó la misma actividad estresante en ambos grupos encontrando resultados diversos, ocasionados por la diferencia en la percepción del estrés por cada individuo. ${ }^{21}$

En comparación a este estudio, el cual se centró en visualizar cómo el ejercicio disminuye el estrés y que es éste el que influye en la ingesta de alimento, la investigación realizada por la Universidad Brigham Young ${ }^{11}$ se basó en la relación ejercicio-alimentación, y se encontró que realizar actividad física ejerce la influencia de perder el interés en los alimentos, sin embargo, en la población analizada por este estudio el ejercicio no influyó en la 
alimentación pero sí en la disminución del estrés. ${ }^{22-24}$ Sin embargo en la metodología se empleó un tiempo de ejercicio de 15 minutos y en la investigación de la Universi- dad Brigham Young se usó un tiempo de 45 minutos, por lo que es probable que en los resultados el efecto del ejercicio sobre la ingesta alimentaria no fuera evidente.

\section{Conclusiones}

La presencia de estrés no es un factor determinante para el consumo de alimentos dulces, no obstante, la actividad física sí reduce el comportamiento de una persona ante el estrés.

\section{Bibliografía}

1. La Naturaleza del Estrés. España. Sociedad Española para el Estudio de la Ansiedad y el Estrés. Actualizado: 24 Diciembre 2011. Visitado por última vez: 22 Diciembre 2015. Disponible en: http://pendientedemigracion.ucm.es/info/seas/estres_lab/el_estres. $\mathrm{htm}$.

2. Seligman, M. E. P. La Auténtica Felicidad/Authentic Happiness (Spanish Edition). Ediciones B. 2007.

3. Vallejo-Ruiloba J. Introducción a la psicopatología y la psiquiatría. $7^{a}$ edición. Elsevier/Masson. Barcelona. 2011.

4. Díaz J. Estrés alimentario y salud laboral vs. estrés laboral y alimentación equilibrada. Med. segur. trab. 2007; 58: 93-99.

5. Tébar-Massó FJ, Garaulet-Aza M, García-Prieto MD. Regulación del apetito: nuevos conceptos. Rev Esp Obes. 2003; 1(1): 13-20.

6. Depresión a Actividad Física. Argentina. Sociedad Argentina de Medicina del Estrés. Actualizado: 10 Marzo 2013. Visitado por última vez: 22 Diciembre 2015. Disponible en: http://www. sames.org.ar/index.php\%3Foption\%3Dcom_co ntent \% 26view\% 3Darticle \% 26id\% 3D51: depr esion-actividad-fisica\% 26catid\% 3D1:articulosnuevos\% 26Itemid\%3D11.

7. Voet $D$, Voet J, Pratt C. Fundamentos de Bioquímica. 2a Edición. Ed. Panamericana. Madrid. 2007.

8. Pritchard LE, Turnbull AV, White A. Pro-opiomelanocortin processing in the hypothalamus: impact on melanocortin signalling and obesity. J Endocrinol. 2002;172(3):411-21.

9. Ekman, P. Darwin's Compassionate View of Human Nature. JAMA, 2010; 303(6): 557-558.

10. Mahan KL, Escott-Stump S, Raymond JL. Krause Dietoterapia. 13a Edición. Ed. Elsevier. Barcelona. 2013.

11. Hanlon B, Larson MJ, Bailey BW, LeCheminant JD. Neural response to pictures of food after exercise in normal-weight and obese women. Medicine and Science in Sports and Exercise. 2012 May;44 (10):1864-1870.

12. Martins C, Robertson MD, Morgan LM. Effects of exercise and restrained eating behavior on appetite control. Proc Nutr Soc. 2008; 67: 28-41.

13. McLaughlin A. Demystifying Intense Desires for Certain Foods. 2013.

14. López A, Rondón J, Alfano S, Cellerino C. Relaciones entre esquemas tempranos inadaptados y afectividad positiva y negativa. Ciencias Psicológicas. 2012; 6 (2): 149-173.

15. Taylor $A H, H$ wajung $O$. Brisk walking reduces ad libitum snacking in regular chocolate eaters during a workplace simulation. Appetite. 2012; 58: 387-392.

16. Vicens $P$, Pueyo AA. Procedimientos de inducción del estado de ánimo y personalidad. Rev Psicol Gral Aplic. 1997; 50(1): 145-157.

17. Del-Pino-Sedeño T, Peñate W, Bethencourt JM. La Escala de Valoración del Estado de Ánimo (EVEA): análisis de la estructura factorial y de la capacidad para detectar cambios en estados de ánimo. Anál Modif Cond. 2012; 36: 153-154.

18. Sanz J. Un instrumento para evaluar la eficacia de los procedimientos de inducción del estado de ánimo: la "escala de valoración del estado de ánimo" (EVEA). Anál Mod Cond. 2001; 27(111).

19. Sanz J. Escala de Valoración del Estado de Ánimo (EVEA). Facultad de Psicología, Universidad Complutense de Madrid. 1993.

20. Godoy $F$, Vega $M$. Inducción de estados de ánimo mediante el método de Velten: una revisión de la literatura. Psicothema 1992; 4(1): 101-111.

21. Marcus C. El Estrés y la Percepción. Huffpost Voces. 2012. Visitado por última vez: 30/12/15. Disponible en: http://voces.huffingtonpost.com/cesar-leomarcus/estres-percepcion_b_1558539.html.

22. Martínez A, Godoy F. La "Escala de Balance Afectivo». Propiedades psicométricas de un instrumento para la medida del afecto positivo y negativo en población española. Clínica y Salud. 2008; 19(2): 157-189.

23. Blundell JE, King NA. Exercise, appetite control, and energy balance. Nutrition. 2000; 16(7-8): 519-522.

24. López-Pell AF, Rondón JM, Alfano SM, Cellerino C. Relaciones entre esquemas tempranos inadaptados y afectividad positiva y negativa. Cienc Psicol. 2012; 6(2): 149-173. 